\title{
Does Food Addiction Exist? A Phenomenological Discussion Based on the Psychiatric Classification of Substance-Related Disorders and Addiction
}

\author{
Özgür Albayrak Sebastian Mathias Wölfle Johannes Hebebrand \\ Department of Child and Adolescent Psychiatry, LVR-Klinikum Essen, University of Duisburg-Essen, \\ Essen, Germany
}

\author{
Key Words \\ DSM-V $\cdot$ Eating disorder $\cdot$ Addiction $\cdot$ Substance-related disorder
}

\begin{abstract}
The relationship between overeating, substance abuse and (behavioral) addiction is controversial. Medically established forms of addiction so far pertain to substance use disorders only. But the preliminary Diagnostic and Statistical Manual for Mental Disorders V (DSM V) suggests replacing the previous category 'Substance-Related Disorders' with 'Addiction and Related Disorders', thus for the first time allowing the diagnosis of behavioral addictions. In the past psychiatrists and psychologists have been reluctant to systematically delineate and classify the term behavioral addiction. However, there is a broad overlap between chemical and behavioral addiction including phenomenological, therapeutic, genetic, and neurobiological aspects. It is of interest to point out that the hormone leptin in itself has a pronounced effect on the reward system, thus suggesting an indirect link between overeating and 'chemical' addiction. Thus, leptin-deficient individuals could be classified as fulfilling criteria for food addiction. In our overview we first review psychological findings in chemical (substancebased) and subsequently in behavioral addiction to analyze the overlap. We discuss the diagnostic validity of food addiction, which in theory can be chemically and/or behaviorally based.
\end{abstract}

Copyright (c) 2012 S. Karger GmbH, Freiburg

\section{Introduction}

The relationship between overeating, substance abuse and behavioral addiction is controversial. Some researchers have argued for an integration of overeating into substance use disorders [e.g. 1, 2]; others suggest replacing increased food intake associated with 
Table 1. ICD-10 F10-19 as mental and behavioral disorders due to psychoactive substance use [5]
Table 2. Subdivided substancerelated disorders in ICD-10 and DSM-IV [5-7]

$\begin{array}{ll}\text { F10 } & \text { Alcohol } \\ \text { F11 } & \text { Opiods } \\ \text { F12 } & \text { Cannabinoids } \\ \text { F13 } & \text { Sedatives or hypnotics } \\ \text { F14 } & \text { Cocaine } \\ \text { F15 } & \text { Other stimulants including caffeine } \\ \text { F16 } & \text { Hallucinogens } \\ \text { F17 } & \text { Tobacco } \\ \text { F18 } & \text { Volatile substances } \\ \text { F19 } & \text { Multiple drug use and use of other psychoactive substances }\end{array}$

\begin{tabular}{ll}
\hline ICD-10 & DSM-IV \\
\hline Acute intoxication & substance intoxication \\
Harmful use & substance abuse \\
Dependence syndrome & substance dependence \\
Withdrawal state & substance withdrawal \\
\hline
\end{tabular}

obesity or eating disorders as a behavioral addiction [3]. An integration into substance use disorders implies a form of chemical addiction for which there is currently only insufficient evidence; a defined chemical in everyday food that can unequivocally induce addiction via binding to specific central nervous system receptors has not been discovered. However, there is evidence to suggest that food addiction can be viewed as a specific form of behavioral addiction in a subgroup of obese individuals. In the following, we discuss diagnostic issues of both substance use disorders and addictive behavioral disorders mainly highlighting their clinical features. Other articles within this special issue will describe the neurobiological features of food addiction.

\section{Classification of Chemical (Substance) Addiction}

The medically established forms of addiction pertain to substance use disorders. The National Institute on Drug Abuse [4] provides the following definition: 'Addiction is defined as a chronic, relapsing brain disease that is characterized by compulsive drug seeking and use, despite harmful consequences'. In Chapter V 'Mental and Behavioral Disorders' of the International Statistical Classification of Diseases and Related Health Problems, 10th Revision (ICD-10; World Health Organization, 1992 [5]) 'Mental and Behavioral Disorders due to Psychoactive Substance Use' (F10-F19) constitute one of the ten major diagnostic categories. ICD-10 specifically refers to mental and behavioral disorders (see table 1). In the second frequently used psychiatric classification scheme termed Diagnostic and Statistical Manual of Mental Disorders (DSM), 4th Edition, Text Revised (DSM-IV-TR), published by the American Psychiatric Association (APA) in 2000 [6], 'Substance-Related Disorders' also represent one of the major diagnostic categories. According to both classification schemes each substancerelated disorder is subdivided into major clinical states (table 2; see table 3 and table 4 for the respective DSM-IV classification criteria). The symptoms of intoxication and withdrawal can differ by substance as can the physical and psychological consequences of substance use. 
Table 3. DSM IV-TR criteria for substance abuse [7]

A. A maladaptive pattern of substance use leading to clinically significant impairment or distress, as manifested by one (or more) of the following, occurring within a 12-month period:

1) Recurrent substance use resulting in a failure to fulfill major role obligations at work, school, or home (e.g., repeated absences or poor work performance related to substance use; substancerelated absences, suspensions, or expulsions from school; neglect of children or household)

2) Recurrent substance use in situations in which it is physically hazardous (e.g., driving an automobile or operating a machine when impaired by substance use)

3) Recurrent substance-related legal problems (e.g., arrests for substance-related disorderly conduct)

4) Continued substance use despite having persistent or recurrent social or interpersonal problems caused or exacerbated by the effects of the substance (e.g., arguments with spouse about consequences of intoxication, physical fights)

B. The symptoms have never met the criteria for Substance Dependence for this class of substance

Table 4. DSM IV-TR criteria for substance dependence [7]

A. Maladaptive pattern of substance use, leading to clinically significant impairment or distress, as manifested by three (or more) of the following, occurring at any time in the same 12 -month period:

1) Tolerance, as defined by either of the following:

a) a need for markedly increased amounts of the substance to achieve intoxication or desired effect

b) markedly diminished effect with continued use of the same amount of the substance

2) withdrawal, as manifested by either of the following:

a) the characteristic withdrawal syndrome for the substance (refer to Criteria A and B of the criteria sets for

Withdrawal from the specific substances

b) the same (or a closely related) substance is taken to relieve or avoid withdrawal symptoms

3) The substance is often taken in larger amounts or over a longer period than was intended

4) There is a persistent desire or unsuccessful efforts to cut down or control substance use

5) A great deal of time is spent in activities necessary to obtain the substance (e.g., visiting multiple doctors or driving long distances), use the substance (e.g., chain smoking), or recover from its effects

6) Important social, occupational, or recreational activities are given up or reduced because of substance use

7) The substance use is continued despite knowledge of having a persistent or recurrent physical or psychological problem that is likely to have been caused or exacerbated by the substance (e.g., current cocaine use despite recognition of cocaine-induced depression, or continued drinking despite recognition that an ulcer was made worse by alcohol consumption)

Specify if:

With physiological dependence: evidence of tolerance or withdrawal (i.e., either item 1 or 2 is present).

Without physiological dependence: no evidence of tolerance or withdrawal (i.e., neither item 1 nor 2 is present). 
Table 5. Preliminary DSM-5 criteria for substance use disorder [8]

A. A maladaptive pattern of substance use leading to clinically significant impairment or distress, as manifested by 2 (or more) of the following, occurring within a 12 -month period:

1) Recurrent substance use resulting in a failure to fulfill major role obligations at work, school, or home (e.g., repeated absences or poor work performance related to substance use; substancerelated absences, suspensions, or expulsions from school; neglect of children or household)

2) Recurrent substance use in situations in which it is physically hazardous (e.g., driving an automobile or operating a machine when impaired by substance use)

3) Continued substance use despite having persistent or recurrent social or interpersonal problems caused or exacerbated by the effects of the substance (e.g., arguments with spouse about consequences of intoxication, physical fights)

4) Tolerance, as defined by either of the following:

a) a need for markedly increased amounts of the substance to achieve intoxication or desired effect

b) markedly diminished effect with continued use of the same amount of the substance (note: tolerance is not counted for those taking medications under medical supervision such as analgesics, antidepressants, anti-anxiety medications or beta-blockers)

5) Withdrawal, as manifested by either of the following:

a) the characteristic withdrawal syndrome for the substance (refer to criteria A and B of the criteria sets for withdrawal from the specific substances)

b) the same (or a closely related) substance is taken to relieve or avoid withdrawal symptoms (note: withdrawal is not counted for those taking medications under medical supervision such as analgesics, antidepressants, anti-anxiety medications or beta-blockers)

6) The substance is often taken in larger amounts or over a longer period than was intended

7) There is a persistent desire or unsuccessful efforts to cut down or control substance use

8) A great deal of time is spent in activities necessary to obtain the substance, use the substance, or recover from its effects

9) Important social, occupational, or recreational activities are given up or reduced because of substance use

10) The substance use is continued despite knowledge of having a persistent or recurrent physical or psychological problem that is likely to have been caused or exacerbated by the substance

11) Craving or a strong desire or urge to use a specific substance

Severity specifiers:

Moderate: 2-3 criteria positive.

Severe: 4 or more criteria positive.

Specify if:

With physiological dependence: evidence of tolerance or withdrawal (i.e., either item 4 or 5 is present). Without physiological dependence: no evidence of tolerance or withdrawal (i.e., neither item 4 nor 5 is present).

DSM-V [7] will replace the current DSM version (DSM-IV-TR) in 2013; currently different workgroups are discussing how to best categorize and operationalize mental disorders and their criteria as based on current empirical findings. After extensive discussions of the word 'addiction', the DSM-V Substance Use Disorders Workgroup has proposed to tentatively re-title the previous category Substance-Related Disorders with Addiction and Related Disorders [8]. The use of the word 'dependence' is now limited to physiological dependence, which is a normal response to repeated doses of many drugs and medications. If appropriate, medical treatment with prescribed drugs entails tolerance and/or withdrawal 
symptoms; these are not to be counted for the diagnosis of substance use disorder. Importantly, the DSM-V Substance Use Disorders Workgroup recommends combining abuse and dependence into a single disorder of graded clinical severity to be termed Substance Use Disorder, with two criteria required to make a diagnosis (table 5). This recommendation was, among other factors, based on problems differentiating abuse from dependence and the lower reliability of the DSM-IV diagnosis Substance Abuse. The current DSM-IV abuse and dependence criteria can be considered to form a unidimensional structure, with abuse and dependence criteria interspersed across the severity spectrum [8].

As illustrated by the discussion within the DSM V Substance Use Disorders Workgroup, classification of psychiatric disorders is subject to change reflecting advances in empirical findings. In the first edition of the APA's Diagnostic and Statistical Manual of Mental Disorders (1952) [9], alcohol and drug abuse were grouped under Sociopathic Personality Disturbances, which were considered to result from deeper psychological disorders or moral weakness. Over the past 60 years we have witnessed the medicalization of addiction to the two major legal (nicotine and alcohol) and all illegal drugs. Thus, the biomedical concept of addiction as underlying excessive use of nicotine or alcohol has in itself led to the delineation of the respective substance use disorders with tremendous implications for the societal perception of these addictions, their treatment, direct and indirect health care costs, and prevention. The medicalization was, among other factors, boosted by the realization of the dire medical consequences of nicotine and alcohol dependency. Efforts of for example the tobacco industry to negate or mitigate the risks of smoking-induced diseases such as lung cancer and cardiovascular disorders are well known; the influence of the tobacco industry on policy is ongoing [10]. Accordingly, understanding how corporations influence policy therefore forms an essential part of public health research into addictions to legal drugs [11].

\section{Classification of Behavioral Addiction}

The term behavioral addiction has more recently been invoked; it has as yet not gained official recognition in medicine: Thus, neither ICD-10 nor DSM-IV-TR includes a respective diagnostic category. Psychiatrists and psychologists have been somewhat reluctant to systematically delineate and classify such disorders. We believe that several factors contribute to this reluctance:

i) The recent history of substance use disorders indicates that the transition from the concept of a behavioral problem as representing a personal flaw to that of an addictive disorder takes time; the process requires both a discussion and merging consensus within medicine and society as a whole.

ii) There is a general reluctance to medicalize and even more so to psychiatrize addictive behaviors, because they can be viewed as partially representing the extreme end of quantitative distributions of time spent pursuing everyday behaviors. Thus, the delineation of distinct disorders requires the definition of threshold or cut-off criteria. In contrast to chemical addiction, the intake of a specific substance, which can readily be verified (i.e., determination of concentrations of diverse drugs and/or their metabolites in serum and urine), is not required. Obviously, soft cut-offs for such disorders would result in high percentages of the population fulfilling the diagnostic criteria for the respective behavioral addiction disorders. Such individuals would then be eligible for a diagnostic evaluation and treatment potentially entailing high costs to national health care systems.

iii) Similar to the situation for legal drugs, the respective behaviors are pursued by a high percentage of the population, thus entailing difficulties at both the individual and societal levels to perceive quantitatively excessive behaviors as disorders. It is difficult to 
Table 6. Proposed DSM V criteria for pathological (disordered) gambling within the new diagnostic category addiction and related disorders [8]

Pathological (disordered) gambling within the new diagnostic category addiction and related disorders

A. Persistent and recurrent maladaptive gambling behavior as indicated by five (or more) of the following:

1) Is preoccupied with gambling (e.g., preoccupied with reliving past gambling experiences, handicapping or planning the next venture, or thinking of ways to get money with which to gamble

2) Needs to gamble with increasing amounts of money in order to achieve the desired excitement

3) Has repeated unsuccessful efforts to control, cut back, or stop gambling

4) Is restless or irritable when attempting to cut down or stop gambling

5) Gambles as a way of escaping from problems or of relieving a dysphoric mood (e.g., feelings of helplessness, guilt, anxiety, depression)

6) After losing money gambling, often returns another day to get even ('chasing' one's losses)

7) Lies to family members, therapist, or others to conceal the extent of involvement with gambling

8) Has jeopardized or lost a significant relationship, job, or educational or career opportunity because of gambling

9) Relies on other to provide money to relieve a desperate financial situation caused by gambling

B. The gambling behavior is not better accounted for by a Manic Episode

appreciate that some individuals who over-engage in specific activities can be seriously functionally impaired in their daily lives.

iv) Modern technologies and media with their easy access account for some of the 'behavioral addictions' (internet) or greatly facilitate them (internet based access to pornographic websites). As such, the problem of their excessive use is rather new and rapidly developing into novel directions; research accordingly lags well behind in comparison to that into substance use disorders.

v) Over-engagement in specific activities is frequently encountered in different psychiatric disorders, which are not considered within the realm of addiction. For example, excessive gambling or internet use may appear within the context of a major depressive episode or an obsessive compulsive disorder; accordingly, the excessive behavior appears to be a symptom or epiphenomenon of the underlying disorder. One view posits addictive behavioral disorders as lying along an impulsive-compulsive spectrum, with some classified as impulse control disorders [12].

The DSM-V Substance Use Disorders Workgroup [8] has recently proposed that the diagnosis of Pathological (Disordered) Gambling (table 6) be reclassified from ImpulseControl Disorders Not Elsewhere Classified' to the novel category Addiction and Related Disorders [13]. Pathological (Disordered) Gambling was judged as having commonalities in clinical expression, etiology (including genetics), comorbidity, physiology, and treatment with Substance Use Disorders, thus warranting this reclassification [e.g. 14, 15]. This proposal indicates a crucial turning point in the official psychiatric conceptualization of this disorder, which is additionally accompanied by the renaming of the diagnostic category. Currently, pathological gambling is to be the only behavioral addictive disorder within the novel DSM V diagnostic category 'Addiction and Related Disorders'. However, this re-classification will undoubtedly boost research and discussions as to the delineation of additional behavioral addictions within this diagnostic category.

Addiction can be defined as an abnormal tolerance to and dependence on something that is psychologically or physically habit-forming [16]. Behavioral addiction implies the 
continued involvement with an activity despite the negative consequences associated with it; pleasure and enjoyment would have originally been sought, however over a period of time involvement with the activity is needed to feel normal [17]. Accordingly, the respective activities have an addictive potential (e.g. gambling, internet, computer games, work, exercise, sexual activity, overeating), some of which pertain to natural homeostatic needs (e.g. eating). A behavior that can function both to produce pleasure and to provide relief from internal discomfort is pursued in a pattern characterized by i) recurrent failure to control the behavior (powerlessness) and ii) continuation of the behavior despite significant negative consequences (unmanageability) [15]. The terms that are used to characterize an addictive disorder are 'dependence' and 'compulsion'. Dependence involves a repetitive pattern of behavior that aims to achieve a pleasurable internal state via gratification of needs. In the terminology of learning and behavior theory, the process by which dependence gratification motivates behavior is termed positive reinforcement. Compulsion involves an attempt to evade or avoid an unpleasant/aversive internal state (e.g. anxiety, grief, guilt, shame, rage). This corresponds to a negative reinforcement paradigm, whereby negative consequences are roughly taken into account. Among the distinguishing features of addictive disorders is this combination of gratification and escape from internal discomfort. Hence, the concept of addiction represents a synthesis of dependence and compulsion [15].

\section{Overlap between Chemical and Behavioral Addiction}

What are the common features of behavioral and chemical addiction? It is primarily the concept of an underlying addictive process, which relates to and affects the individual's life, and brings together all the different sorts of addictive behaviors. The underlying addictive process is in essence the compulsive dependence on an (apparently self-initiated and selfcontrolled) external action in order to regulate the internal state. Behavioral and substance addicts resemble each other: Both have the urge to engage in their behavioral routine; they feel discomfort if prevented from completing it resulting in craving and withdrawal symptoms. Some withdrawal symptoms (e.g. anxiety) are identical across certain behavioral and chemical addicts while others (e.g. runny eyes and sneezing in opiate withdrawal) are substance-specific $[17,18]$.

Donegan et al. [19] proposed seven properties which addictive substances or activities (including food and gambling) have in common:

i) Ability of the substance/activity to act as an instrumental re-enforcer.

ii) Acquired tolerance - repeated use can result in reduced effectiveness of the substance/activity.

iii) Development of dependence with repeated use; if the substance is not available or the activity cannot be pursued withdrawal symptoms ensue that motivate further use.

iv) Affective contrast: The substance/activity tends to produce an initial positive affective state (euphoria), which is then followed by an opposing negative state (dysphoria).

v) Ability of the substance/activity to act as an effective Pavlovian unconditioned stimulus.

vi) Ability of various states (general arousal, stress, pain, mood) to influence substance use or engagement in the respective activity.

vii) Behavioral and chemical 'addicts' may become prompted by both internal cues, such as boredom, depression or well-being, and external cues, such as places or people. Individual cues will vary depending on both the individual and the chemical/behavioral type of addiction. 


\section{Therapeutic Considerations}

From a therapeutic point of view, each of the various behavioral and chemical addictions has its own particular pattern of relapse management. Internet addicts need to learn how to engage in relationships despite their social avoidance; teenage smokers should acquire the skill of saying 'no' without giving offence or losing status; and overeaters should learn how to use different coping skills to reduce caloric intake. But the internal cues that condition to addiction seem similar across the various disorders. Addicts of all kinds are more likely to indulge when they feel miserable, anxious, bored, and/or stressed. One treatment aspect common to all addiction disorders is that patients need to learn to sense what feelings/ conditions give rise to or increase their craving and to come up with alternative strategies to circumvent intake of a substance or their indulging in the respective activity. If the addiction is long standing and keeps the patient occupied for large parts of the day, such an individual will have to relearn how to make use of regained time $[18,19]$.

\section{Psychiatric Comorbidity}

In patients with substance use disorders, psychiatric comorbidity is the rule rather than exception. Psychiatric disorders frequently precede the development of the addiction, but can also develop after its onset. Models of bidirectional relationships or contemporaneous combination of risk factors are part of this complex discussion [20]. Mood, anxiety, and conduct disorders represent the most frequent comorbidities. The likelihood for comorbidity of depression or anxiety disorder in adults with drug/alcohol dependency is 2-3 times higher than in the general population [21]. Similarly, a broad range of psychiatric comorbidities apply to behavioral addictions. For example, pathological internet use or internet dependent individuals have elevated rates of depression or attention deficit/hyperactivity disorder (ADHD) [22]. The latter disorder also occurs more frequently in substance use disorders.

\section{Genetic Overlap}

Family and twin studies have estimated that genetic contributions account for up to $60 \%$ of the variance in the risk for substance addictions $[23,24]$. Similarly robust genetic contributions in the magnitude of $35-54 \%$ have been found for pathological gambling (PG) [25]. In light of the substantial body of evidence from family, twin, and adoption studies indicating a genetic component as underlying all addiction disorders [26], it is of interest to focus on studies providing evidence for a common genetic diathesis of both chemical and behavioral addictions. Based on assessment of lifetime histories of PG and alcohol dependence the extent to which environmental and genetic risk for PG was shared with alcohol dependence has been quantified: A significant proportion of the risk for subclinical PG (12-20\% of genetic and 3-8\% of environmental factors) was accounted for by the risk of alcohol dependence [27]. Genetic factors also play a role in the personality traits and behavioral disorders that are associated with increased experimentation with drugs (i.e., initiation): novelty seeking, impulsiveness, response to stress, but also psychiatric diagnoses like ADHD, conduct disorder, antisocial personality disorder, mood and anxiety disorders [26].

\section{Neurobiological Overlap}

Neurobiological models for developing a dependency or addiction with a chemical substance or with a behavioral character tend to identify a common cause [22,28]. Different neurotransmitters (e.g. dopamine, glutamate, norepinephrine) have an influence on the development or status of addiction or dependency. Dopaminergic neurons, originating from the ventral tegmental area (VTA) synapse within the nucleus accumbens (NAcc), form the 
major arm of the brain's natural reward system, which mediates the rewarding effects of behaviors such as food intake, social interactions, and sex. [29, 30]. Another neurotransmitter, glutamate, as the most abundant excitatory physiological neurotransmitter is implicated in motivational processes, drug addiction, and impulse control disorders [31]. Other studies indicate that levels of glutamate within the NAcc mediate reward seeking behavior. In addition, norepinephrine influences multiple brain functions including arousal, attention, learning, stress response, and subjective rewarding effects [32]. However, the reward-reinforcement circuit is not solely important for addictive behaviors. It has also been implicated in other psychiatric conditions (e.g. schizophrenia) [33].

Leptin, a major signal of long-term energy balance, modulates neural activation in key striatal regions, suggesting that the hormone acts on neural circuits governing food intake to diminish the perception of food reward, while enhancing the response to satiety signals generated during food consumption. Leptin appears to play multiple roles in the mesolimbic dopamine system. It promotes a complex set of changes in the mesolimbic dopamine system against addictive properties. Thus, leptin in itself influences the reward system [34]. Leptin resistance results from chronic overstimulation of the anorexigenic adipocytic leptin signal in the case of obesity, potentially leading to weakened signal for the reduction of the perception of food reward; leptin's anorexigenic signal is attenuated.

Continuous overeating can be viewed as an addictive behavior. Both leptin and ghrelin are hormones that influence the hypothalamic regulation of food intake and energy homeostasis and promote satiety and hunger, respectively. Several studies have documented that ghrelin also acts on dopaminergic reward system components, e.g. VTA and NAcc. Interestingly, both hormones have been invoked to play a role in alcohol and cocaine craving [3538]. Therefore, these hormones may well be considered as forming the biological link between 'chemical' and behavioral food addiction.

Another factor that influences the reward system is stress. It acts on the hypothalamicpituitary-adrenal (HPA) axis via release of corticotrophin-releasing factor (CRF), which has been shown to stimulate the reward system components VTA, NAcc, and dopaminergic transmission. Physiologically, CRF release is regulated via a negative-feedback loop upon cortisol production. Chronic stress leads to an overproduction of CRF and cortisol, thus abolishing the negative-feedback loop [39]. It is hypothesized that a dysregulation of the HPA axis leads to negative reinforcement drives and potentially increases addiction risk [40, 41].

\section{Obesity}

Obesity is an exceedingly complex disorder, which evidently requires an environment that promotes a high energy intake and/or a low level of physical activity. In societies with diverse, tasty, cheap, and readily available foods a genetic predisposition is presumably also required to put on excess weight. The heritability of body weight is high - it is assumed that $50 \%$ or more of the variance of the BMI in the general population can be explained by genetic factors. However, currently known polygenic loci only explain a small percentage of the BMI variance $[42,43]$. Overeating implies an energy intake in excess of energy expenditure. Individuals with a low resting energy expenditure and/or a low physical activity level can overeat and thus gain weight despite intake of normal portion sizes. In many obese individuals excess weight gain occurred over prolonged periods of time; accordingly, obesity rates in young adults are substantially lower than in middle-aged adults [44-48]. If for example the daily energy excess is a mere $20 \mathrm{kcal}$, relative body weight will increase slowly over the life span [45]. Reference to addiction as an explanation for the common occurrence 
Table 7. Proposed DSM-5 criteria for binge eating disorder [8]

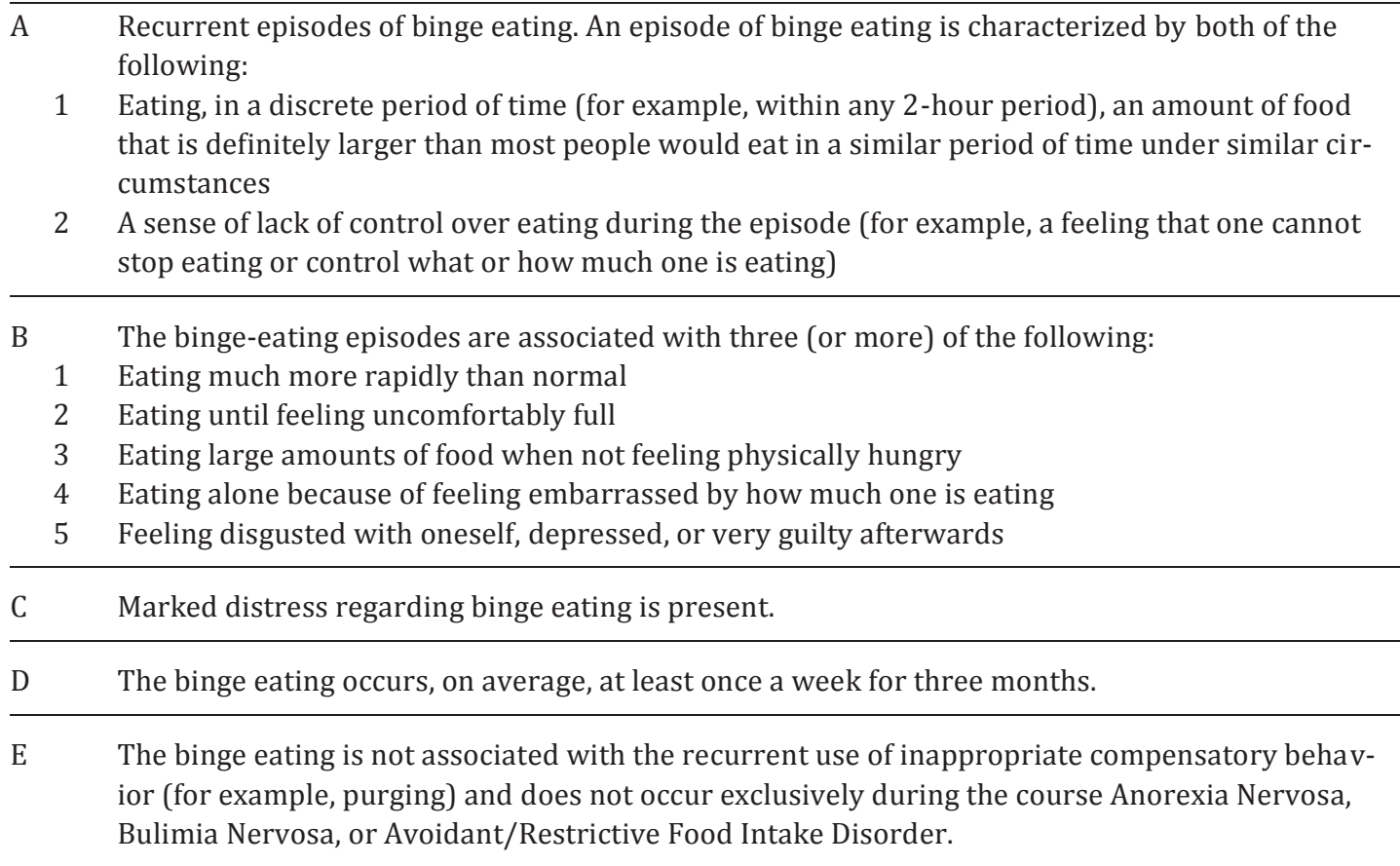

of this type of obesity seems totally inappropriate. Addiction also cannot readily be inferred as an explanation for the well-known difficulties of maintaining weight loss after a diet. Renewed weight gain largely results from physiological adaptations, including increased appetite and hunger and a reduced energy expenditure as a reaction to a prolonged curtailment of energy intake. Psychological health and long-term behavior monitoring characterize those individuals who successfully maintain a reduced body weight [49].

Due to the insight we have gained into regulation of food intake and body weight, it is difficult to draw a line between food addiction and a biologically driven increased appetite or hunger. Thus, leptin-deficient patients show food craving, withdrawal, and overeat from infancy on [50]; their behavior throughout the day centers on seeking and ingesting food. They would obviously fulfill criteria for a diagnosis of a substance use disorder (table 7) except for the fact that their addiction applies to food in general, and not to a specific ingredient, substance, or 'chemical'.

Mutations in the melanocortin receptor 4 gene have also been found to result in overeating [51], albeit to a lesser extent than in leptin-deficient subjects. Potentially, polygenic effects also add up and entail an increased appetite/hunger, overeating, and the development of obesity. If due to genetic or other reasons (e.g. hypoxia entailing brain damage, brain tumor) appetite/hunger of a human is in the uppermost range of the normal distribution, this could render his/her reward system particularly dependent on the input of the neuropeptides, neurotransmitters, and hormones involved the regulation of eating behavior. As such, 'addictive behavior' could ensue.

Currently, bulimia nervosa (BN) and binge eating disorder (BED), which will presumably receive the status of a formal eating disorder in DSM V [52], are the only psychiatric disorders which have features that bear resemblance to addiction (see table 7 for proposed DSM-5 diagnostic criteria for BED). The core features of these eating disorders are based on binge eating episodes associated with the subjective experience of a lack of control. However, in 
contrast to patients with BED, counter-regulation (e.g. purging) is a prominent feature of BN $[7,53]$. In clinically ascertained BED patients obesity is common. However, the association with obesity is diluted in the community; according to an epidemiological study, only two thirds of BED subjects were obese [for review see 53,54]. Other types of aberrant eating behaviors such as night eating and grazing have been described, which can potentially be viewed within the context of addiction. The current classification scheme DSM-IV-TR however only allows the diagnosis of an Eating Disorder Not Otherwise Specified for BED and other disordered eating patterns of clinical significance. It is of interest to discuss implications of classifying BED as a form of addictive behavior in DSM-V. This would encourage researchers to investigate more extensively the overlap with addiction and to apply therapeutic principles, which are more commonly used in addictive medicine [33].

The excessive overconsumption of palatable foods and fluids as seen in binge eating episodes may be indicative of an underlying neurobiological process similar to that seen in addiction $[55,56]$. This conclusion is drawn from a growing body of evidence that substancerelated disorders and obesity both share common neural mechanisms [57]. Thus, in obese rats, hypofunctionality of the reward system occurs due to a blunted dopamine transmission in the reward center of the brain following high-caloric excess, which leads to compulsive-like eating in such rodents [58]. These maladaptive behavioral responses in obese rats likely arise from diet-induced deficits in striatal dopamine D2 receptor signaling. Overconsumption of drugs of abuse similarly decreases striatal dopamine D2 receptor density, induces a profound state of reward hypofunctionality, and triggers the emergence of compulsive-like drug-taking behaviors $[59,60]$. Similarly, human imaging studies have shown that obese subjects may have impairments in dopaminergic pathways that regulate neuronal systems associated with reward sensitivity, conditioning, and control [61]. But currently it is unclear if these findings are indicative of predisposing factors or represent the consequence of overeating.

The release of endorphins upon excessive exercise [62] reveals that the general term chemical addiction by itself does not necessarily require the substance to be an exogenous chemical. If endogenous 'chemicals' can be addictive in specific circumstances and/or in thus predisposed individuals, such neural mechanisms may represent a link between drug and behavioral addiction. The link of appetite, hunger, satiation, and satiety with the reward system can be viewed as the basis for the development of addictive eating. Even individuals who overeat despite not being hungry trigger alterations in complex central regulatory systems, which in theory could suffice to initiate and maintain addiction. The respective psychological cues could encompass boredom, perceived stress, a negative mood, and the like. Nevertheless, it should be clearly pointed out that, in light of the absence of an operational definition of food addiction, it is currently not possible to evaluate its validity and reliability as a diagnostic category. It is thus premature to consider food addiction within the psychiatric diagnostic classification systems. Studies are required to precisely delineate the symptoms, associated psychopathology and response to treatments [33].

We have mainly discussed food addiction as a subtype of behavioral addiction. However, because the term 'food' refers to a heterogeneous composite of a variety of dietary components, be it natural nutrients (e.g. fat, sugar) or synthetic food additives (e.g. preservatives), it is critical for the understanding of the nature of the underlying processes pertaining to food addiction to investigate whether these single nutrient components in itself display reinforcing behavioral properties and thus bear the possibility to lead to neurobiological alterations in the reward system, equivalent to substances of abuse such as heroin, cocaine, alcohol, or nicotine. As such, the nutrient component ought to be absorbed in the oro-gastrointestinal tract itself or as a direct metabolite cross the blood-brain barrier and unfold its reinforcing effects via activation of the reward system. Indeed, various animal studies 
center on the effects of sugar on the mesolimbic dopamine projections from the VTA to the NAcc which is implicated in reinforcement functions [63] and attributed to display an incentive effect on the motivation in the process of getting addicted [64]. Extracellular dopamine in the NAcc increases after intake of drugs that are abused $[65,66]$. Rats intermittently food-deprived and fed with $10 \%$ sucrose dilution and chow develop a binge eating behavior. Similar to the intake of drugs, these rats release extracellular dopamine in the NAcc, every time they binge sugar (i.e., sucrose), whereas this dopamine response upon sugar feeding is blunted in control animals fed ad libitum sugar and chow [67]. Intermittent intake of $25 \%$ aqueous glucose and chow in rats revealed behavioral and neurochemical signs of opioid dependency $[68,69]$.

The aforementioned animal studies used glucose or sucrose with chow in combination with intermittent food deprivation. Although these experiments could point toward the addictive potential of sugar, unlike in drugs of abuse, there is no evidence for a specific chemical structure of a nutrient unequivocally leading to neurobiological mechanisms underlying addiction. Excluding studies based on intermittent food deprivation, we are not aware of a series of animal studies, albeit human trials, that repeatedly demonstrated a defined nutrient component with a given chemical structure to cause changes in the reward system similar to those described for drugs. Humans who excessively overeat usually do not continuously resort to a single food molecule or a specific monotonous diet; diets rich in carbohydrates and/or fat contain multiple ingredients.

\section{Food Ingredients}

Obviously, it is extremely difficult to investigate the rewarding properties of a single nutrient component in humans. The term 'food addiction' is mainly used in the context of industrially refined 'highly palatable' food, such as sweet beverages or high-fat diets [1]. This kind of food never contains only one single component. Attempts have been made to establish laboratory procedures to investigate the addictive potential of carbohydrate-rich diets in 'carbohydrate cravers' [69]. It is hypothesized that carbohydrate cravers snack carbohydrate-rich nutrients in a state of depressed or dysphoric mood in order to ameliorate their low affective state, suggesting that carbohydrates lead to an insulin-mediated mechanism, which consequently increases influx of tryptophan into the brain to counterbalance a low brain serotonin level. These experiments [e.g. 70], however, do not overcome methodological flaws and do not point toward a systemic rewarding effect of specific carbohydrates.

Basically, psychologists discriminate two reciprocal and supplementary aspects of reward, 'wanting' and 'liking', with the latter referring to the hedonic rewarding aspect of a substance or a behavior - thought to be attributed to the opioid-system - and the former referring to the incentive sensitization that creates the motivation to seek for the drug or pursue the respective behavior, which is thought to be mediated via the dopaminergic VTA-NAcc circuitry [71]. It seems theoretically plausible, that 'food addiction' might be attributed to the 'wanting' aspect of food reward. Evidently, there is 'wanting' without 'liking', i.e., binge eating is a rather unpleasant experience, where the individual compulsively seeks for and ingests large quantities of food.

While the aforementioned considerations would support the concept of food addiction as a form of behavioral, and not a chemical, addiction, we should be aware of the implications. Generally, any human homeostatic activity which exerts an effect on the reward system would thus qualify as entailing the potential for the development of behavioral addiction. Examples include sex and physical activity. Indeed, both sex and jogging addiction have been described in the psychiatric literature. Such addictions could result from the respective subjects being in the uppermost range of the quantitative distributions of such 
behaviors (strong sex drive, high physical activity), which at an individual level cannot be sufficiently controlled without entailing impairment or harmful consequences. Such addictions could also result via learning from both positive and negative reinforcement of the respective behaviors.

\section{Conclusion and Future Research}

We have critically discussed food addiction in relationship to both chemical and behavioral addiction. Because of the current rather limited evidence of the addictive behavior of specific food ingredients or additives, we currently conclude that food addiction can best be classified as a behavioral addiction at this time. However, because there is not sufficient (i.e., reliable and valid) data on its diagnostic criteria, we would not recommend adding 'food addiction' as a diagnostic entity in DSM-V [33]. The endogenous neuropeptides, neurotransmitters, and hormones, which are released upon ingestion of food, may provide a link between chemical and behavioral addiction. The rewarding properties of food are greater after food deprivation than in satiated organisms. We argue that, because most types of obesity are based on a slight degree of overeating and thus evolve slowly over time, only the combination of clinically significant and regular overeating such as within the context of abnormal eating behavior (currently classified in the category eating disorders) warrants consideration as food addiction. In our opinion, subtypes of obesity that are associated with clinically relevant overeating can be considered with the context of food addiction. Substantially more research into clinically aberrant eating behaviors and patterns and in particular those related to overeating is required to assess if some of the currently poorly described aberrant eating behaviors/disorders cannot be better classified within the recently proposed novel DSM V category Addiction and Related Disorders. As such, the focus should be overeating per se, irrespective of whether it occurs in episodes with or without counterregulation. Additional neurobiological research in both animals and humans is required to strengthen the notion that overeating can be viewed as behavioral addiction. Eating is based on a very complex set of physiological, psychological and neurobiological mechanisms. Visual appearance, oro-sensory sensation, texture of food, the situation in which food is represented, the individual psychological state of mood as well as the individual physiological state of energy and appetite regulation have an influence of how and what humans eat. We conclude that overeating may be viewed as food addiction in a small subgroup of obese individuals.

\section{Disclosure Statement}

The authors declared no conflict of interest.

\section{References}

1 Ifland JR, Preuss HG, Marcus MT, Rourke KM, Taylor WC, Burau K, Jacobs WS, Kadish W, Manso G: Refined food addiction: a classic substance use disorder. Med Hypotheses 2009;72:518-526.

2 Corwin RL, Grigson PS: Symposium Overview - Food Addiction. J Nutr 2009;139:617-619.

3 Corsica JA, Pelchat ML: Food addiction: true or false? Curr Opin Gastroenterol 2010;26:165-169.

4 NIDA: http://www.drugabuse.gov/.

5 WHO: International Statistical Classification of Diseases and Related Health Problems 10th Revision. http:// apps.who.int/classifications/apps/icd/icd10online/. 
Albayrak et al.: Does Food Addiction Exist? A Phenomenological Discussion Based on the Psychiatric Classification of Substance-Related Disorders and Addiction

6 American Psychiatric Association: DSM-IV. www.psych.org/MainMenu/Research/DSMIV.aspx.

7 American Psychiatric Association: DSM-IV-TR: The Current Manual.: www.psych.org/mainmenu/research/ dsmiv/dsmivtr.aspx.

8 American Psychiatric Association: DSM-5. www.dsm5.org/ProposedRevisions/Pages/Substance-RelatedDisorders.aspx.

9 Raines GN: Comment: the new nomenclature. Am J Psychiatry1953;109:548-549.

-10 Smith KE, Fooks G, Collin J, Weishaar H, Mandal S, Gilmore AB: 'Working the System' - British American Tobacco's influence on the European Union Treaty and its implications for policy: An analysis of internal tobacco industry documents. PLoS Med 2010;7:e1000202.

11 Gearhardt AN, Grilo CM, DiLeone RJ, Brownell KD, Potenza MN: Can food be addictive? Public health and policy implications. Addiction 2011;106:1208-1212.

-12 Grant JE, Potenza MN, Weinstein A, Gorelick DA: Introduction to behavioral addictions. Am J Drug Alcohol Abuse 2010;36:233-241.

13 American Psychiatric Association: DSM-V: R 31 Gambling Disorder. www.dsm5.org/ProposedRevisions/Pages/ proposedrevision.asp $x$ ? $r i d=210 \#$.

14 Brewer JA, Potenza MN: The neurobiology and genetics of impulse control disorders: relationships to drug addictions. Biochem Pharmacol 2008;75:63-75.

15 Marks I: Behavioural (non-chemical) addictions. Br J Addict 1990;85:1389-1394.

16 Addiction: $h t t p: / / w o r d n e t w e b . p r i n c e t o n . e d u / p e r l / w e b w n ? s=a d d i c t i o n$.

17 Morrissey J, Keogh B, Doyle L (eds): Psychiatric Mental Health Nursing. Dublin, Gill \& Macmillan, 2008, p 289.

18 Bradley BP: Behavioural addictions: common features and treatment implications Br J Addict 1990;85: 1417-1419.

19 Donegan NH, Rodin J, O’Brien C, Solomon RL: A learning-theory approach to commonalities; in Levison PK, Gerstein DR, Maloff DR (eds): Commonalities in Substance Abuse and Habitual Behavior. Lexington, Lexington Books, 1983, pp 157-235.

-20 Mueser KT, Drake RE, Wallach MA: Dual diagnosis: a review of etiological theories. Addict Behav 1998;23: 717-734.

-21 Grant BF, Stinson FS, Dawson DA, Chou SP, Dufour MC, Compton W, Pickering RP, Kaplan K: Prevalence and co-occurrence of substance use disorders and independent mood and anxiety disorders: results from the National Epidemiologic Survey on Alcohol and Related Conditions. Arch Gen Psychiatry 2004;61:807-816.

-22 Peukert P, Sieslack S, Barth G, Batra A: Internet and computer game addiction. Psychiatr Prax 2010;37: 219-224.

23 Kreek MJ, Nielsen DA, Butelman ER, LaForge KS: Genetic influences on impulsivity, risk taking, stress responsivity and vulnerability to drug abuse and addiction. Nat Neurosci 2005;8:1450-1457.

24 Kreek MJ, Bart G, Lilly C, LaForge KS, Nielsen DA: Pharmacogenetics and human molecular genetics of opiate and cocaine addictions and their treatments. Pharmacol Rev 2005;57:1-26.

-25 Eisen SA, Lin N, Lyons MJ, Scherrer JF, Griffith K, True WR, Goldberg J, Tsuang MT: Familial influences on gambling behavior: an analysis of 3359 twin pairs. Addiction 1998;93:1375-1384.

26 Lachmann HM: An overview of the genetics of substance abuse disorders Curr Psychiatry Rep 2006; 8: 133-143.

-27 Slutske WS, Eisen S, True WR, Lyons MJ, Goldberg J, Tsuang M: Common genetic vulnerability for pathological gambling and alcohol dependence in men. Arch Gen Psychiatry 2000;57:666-673.

28 Potenza MN: The neurobiology of pathological gambling and drug addiction: an overview and new findings. Philos Trans R Soc Lond B Biol Sci 2008;363:3181-3189.

29 Nestler EJ: Is there a common pathway for addiction? Nat Neurosci 2005;8:1445-1449.

-30 Everitt BJ, Robbins TW: Neural systems of reinforcement for drug addiction: from actions to habits to compulsion. Nat Neurosci 2005;8:1481-1489

-31 Kalivas PW, Volkow ND: The neural basis of addiction: a pathology of motivation and choice. Am J Psychiatry 2005;162:1403-1413.

-32 Sofuoglu M, Sewell AR: Norepinephrine and stimulant addiction. Addict Biol 2009;14:119-129.

-33 Moreno C, Tandon R: Should overeating and obesity be classified as an addictive disorder in DSM-5? Curr Pharm Des 2011;17:1128-1131.

34 Opland DM, Leinninger GM, Myers MG Jr: Modulation of mesolimbic dopamine system by leptin. Brain Res 2010;1350:65-70.

-35 Dickson SL, Egecioglu E, Landgren S, Skibicka KP, Engel JA, Jerlhag E. The role of the central ghrelin system in reward from food and chemical drugs. Mol Cell Endocrinol 2011;340:80-87.

-36 Kiefer F, Jahn H, Kellner M, Naber D, Wiedemann K: Leptin as a possible modulator of craving for alcohol. Arch Gen Psychiatry 2001;58:509-510.

37 Kiefer F, Jahn H, Wolf K, Kämpf P, Knaudt K, Wiedemann K: Free-choice alcohol consumption in mice after application of the appetite regulating peptide leptin. Alcohol Clin Exp Res 2001;25:787-789.

-38 Jerlhag E, Egecioglu E, Dickson SL, Engel JA: Ghrelin receptor antagonism attenuates cocaine- and amphetamine-induced locomotor stimulation, accumbal dopamine release, and conditioned place preference. Psychopharmacology (Berl) 2010;211:415-422. 
Albayrak et al.: Does Food Addiction Exist? A Phenomenological Discussion Based on the Psychiatric Classification of Substance-Related Disorders and Addiction

39

$>40$

357.

Koob GF: Neurobiological substrates for the dark side of compulsitivity in addiction. Neuropharmacology 2009;56(suppl1):18-31

42 Hebebrand J, Volckmar AL, Knoll N, Hinney A: Chipping away the 'missing heritability': GIANT steps forward in the molecular elucidation of obesity - but still lots to go. Obes Facts 2010;3:294-303.

43 Speliotes EK, Willer CJ, Berndt SI, Monda KL, et al: Association analyses of 249,796 individuals reveal 18 new loci associated with body mass index. Nat Genet 2010;42:937-948.

-44 Hebebrand J, Bulik CM: Critical appraisal of the provisional DSM-5 criteria for anorexia nervosa and an alternative proposal. Int J Eat Disord. 2011;44:665-678.

45 Weigle DS: Appetite and the regulation of body composition. FASEB J 1994;8:302-310.

46 Hebebrand J: Diagnostic issues in eating disorders and obesity. Child Adolesc Psychiatr Clin N Am 2009;18: $1-16$.

47 Ogden CL, Carroll MD, McDowell MA, Flegal KM: Obesity among adults in the united states- no statistically significant change since 2003-2004. www.cdc.gov/nchs/data/databriefs/db01.pdf.

482000 CDC Growth Charts: United States. www.cdc.gov/growthcharts.

49 G Wing RR, Phelan S: Long-term weight loss maintenance. Am J Clin Nutr 2005;82(1 suppl):222S-225S

50 Montague CT, Farooqi IS, Whitehead JP, Soos MA, Rau H, Wareham NJ, Sewter CP, Digby JE, Mohammed SN, Hurst JA, Cheetham CH, Earley AR, Barnett AH, Prins JB, O’Rahilly S: Congenital leptin deficiency is associated with severe early-onset obesity in humans. Nature 1997;387:903-908.

$\checkmark 51$ Farooqi IS, Keogh JM, Yeo GS, Lank EJ, Cheetham T, 0’Rahilly S. Clinical spectrum of obesity and mutations in the melanocortin 4 receptor gene. N Engl J Med 2003;348:1085-1095.

52 American Psychiatric Association: DSM-V: K 05 Binge Eating Disorder. www.dsm5.org/ProposedRevisions/ Pages/proposedrevision.aspx? rid $=372$.

53 Hebebrand J, Herpertz-Dahlmann B: Diagnostic Issues in eating disorders and obesity. Child Adolesc Psychiatr Clin N Am 2009;18:49-56.

54 Grucza RA, Przybeck TR, Cloninger CR: Prevalence and correlates of binge eating disorder in a community sample. Compr Psychiatry 2007;48:124-131.

55 Mathes WF, Brownley KA, Mo X, Bulik CM: The biology of binge eating. Appetite 2009;52:545-553. Marcus MD, Kalarchian MA: Binge eating in children and adolescents. Int J Eat Disord 2003;34:S47-57. Volkow ND, Wise RA: How can drug addiction help us understand obesity? Nat Neurosci 2005;8:555-560 Johnson PM, Kenny PJ: Dopamine D2 receptors in addiction-like reward dysfunction and compulsive eating in obese rats. Nat Neurosci 2010;13:635-641.

-59 Kenny PJ, Chen SA, Kitamura O, Markou A, Koob GF: Conditioned withdrawal drives heroin consumption and decreases reward sensitivity. J Neurosci 2006;26:5894-5900.

60 Ahmed SH, Kenny PJ, Koob GF, Markou A: Neurobiological evidence for hedonic allostasis associated with escalating cocaine use. Nat Neurosci 2002;5:625-626.

61 Volkow ND, Wang GJ, Baler RD: Reward, dopamine and the control of food intake: implications for obesity. Trends Cogn Sci 2011;15:37-46.

62 Hamer M, Karageorghis Cl: Psychobiological mechanism of exercise dependence. Sports Med 2007;37:477484.

63 Wise RA, Bozarth MA: Brain reward circuitry: four circuit elements 'wired' in apparent series. Brain Res Bull 1984;12:203-208.

-64 Avena NM, Rada P, Hoebel BG: Evidence for sugar addiction: behavioral and neurochemical effects of intermittent, excessive sugar intake. Neurosci Biobehav Rev 2008;32:20-39.

65 Di Chiara G, Imperato A: Drugs abused by humans preferentially increase synaptic dopamine concentrations in the mesolimbic system of freely moving rats. Proc Natl Acad Sci U S A 1988;85(14):5274-5278.

66 De Vries TJ, Shippenberg TS: Neural systems underlying opiate addiction. J Neurosci 2002;22:3321-3325. Rada P, Avena NM, Hoebel BG: Daily bingeing on sugar repeatedly releases dopamine in the accumbens shell. Neuroscience 2005;134:737-744.

68 Colantuoni C, Rada P, McCarthy J, Patten C, Avena NM, Chadeayne A, Hoebel BG: Evidence that intermittent, excessive sugar intake causes endogenous opioid dependence. Obes Res 2002;10:478-488.

-69 Wurtman J, Wurtman R, Berry E, Gleason R, Goldberg H, McDermott J, Kahne M, Tsay R: Dexfenfluramine, fluoxetine, and weight loss among female carbohydrate cravers. Neuropsychopharmacology 1993;9:201210.

70 Spring B, Schneider K, Smith M, Kendzor D, Appelhans B, Hedeker D, Pagoto S: Abuse potential of carbohydrates for overweight carbohydrate cravers. Psychopharmacology (Berl) 2008;197:637-647.

71 Berridge KC: Wanting and liking: observations from the neuroscience and psychology laboratory. Inquiry (Oslo) 2009;52:378. 